\section{Checkpoint Blockade Therapy}

\section{A COMPUTATIONAL SEMI-MECHANISTIC PHARMACOLOGY MODEL OF ATG101, A PD-L1/4-1BB BISPECIFIC ANTIBODY FOR TREATMENT OF SOLID TUMORS AND NHL}

${ }^{1}$ David Flowers*, 'Marc Presler, ${ }^{1}$ Kas Subramanian, ${ }^{1}$ Theresa Yuraszeck, ${ }^{2}$ Hui Yuwen, ${ }^{3}$ Bing Hou, ${ }^{4}$ Dirk Hoenemann. ${ }^{1}$ Applied BioMath, LLC, Concord, MA, USA; ${ }^{2}$ Shanghai Antengene Corporation Limited, Shanghai, China; ${ }^{3}$ Antengene Corporation Co., Ltd, Shanghai, China; ${ }^{4}$ Antengene Pty Ltd, Melbourne, Australia

Background Bispecific antibodies have shown significant promise as therapies for various cancers. ${ }^{1}$ ATG101 is a bispecific antibody that crosslinks tumor-expressed PD-L1 to T-cellexpressed $4-1 \mathrm{BB}$, thereby selectively activating tumor-infiltrating T-cells while inhibiting immune checkpoints. The pharmacologically active complex, which is a trimer consisting of drug bound to both PD-L1 and $4-1 \mathrm{BB}$ and is difficult to measure directly, was predicted using a semi-mechanistic pharmacological model by integrating and calibrating against data from multiple in vitro assays. The model was then used to predict both (1) the number of PD-L1:ATG101:4-1BB trimers formed per T-cell and (2) PD-L1 occupancy, allowing it to quantify 4-1BB-driven activation as well as checkpoint blockade along the PD-1:PD-L1 axis in vivo.

Methods

Model structure A single-compartment in vitro model and a three-compartment in vivo model were built (figure 1). The bispecific molecules could first bind to either 4-1BB or PD-L1 and then crosslink the other receptor to form a trimer.Parameter estimation: The model's binding parameters were calibrated to experimental in vitro data (figure 2). Two crosslinking rates were carried forward to model predictions to capture uncertainty in its value. Other model parameters were estimated from the literature.Model validation: The model was validated by comparing model simulations to data from two in vitro assays (figure 3).In vivo predictions: Simulations of ATG101 in human solid tumor patients were performed using the calibrated binding parameters and standard antibody pharmacokinetic parameters. Simulations varied the crosslinking rate and number of PD-L1 receptors per tumor cell to capture important sources of uncertainty and variability. Single IV bolus doses ranging from 0.001 to $30 \mathrm{mg} / \mathrm{kg}$ were simulated.

Results The model predicts trimer formation exhibits a nonmonotonic dose response and that doses between 0.3 and 3 $\mathrm{mg} / \mathrm{kg}$ maximize trimer formation. Simulations predict that ATG101 can sustain greater than $80 \%$ and $70 \%$ trimer formation and $90 \%$ and $95 \%$ PD-L1 occupancy in the tumor at a dose of $2 \mathrm{mg} / \mathrm{kg}$ and $3 \mathrm{mg} / \mathrm{kg}$, respectively, once every three weeks (figure 4).
A

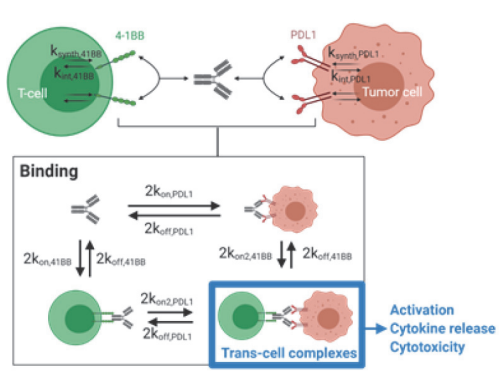

B

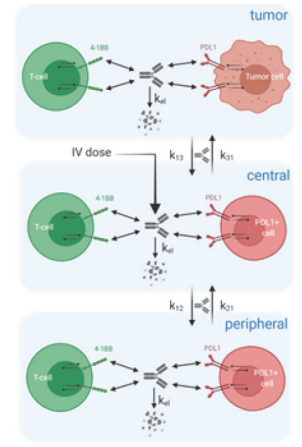

Abstract 227 Figure 1 Model structure diagram for (A) the onecompartment in vitro model and (B) the three-compartment in vivo model
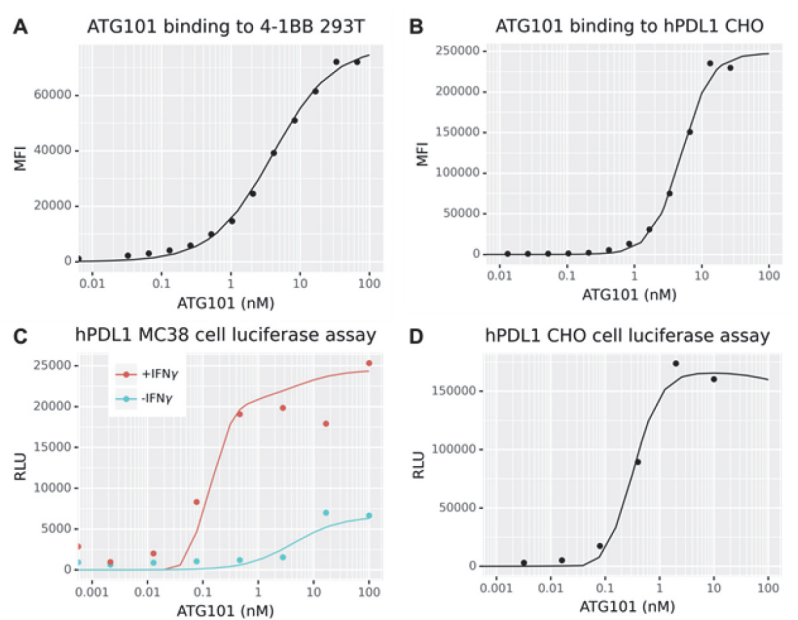

Abstract 227 Figure 2 Comparison of in vitro model simulations to data used for calibration. The 4-1BB and PD-L1 monovalent binding affinities were calibrated to (A) 4-1BB expressing 293T cell binding data and (B) PD-L1-expressing CHO cell data. The crosslinking rate was calibrated to luciferase assays measuring NFKB signaling via 4-1BB crosslinking. In these assays, 4-1BB-expressing 293T cells were incubated with ATG101 and either (C) unstimulated or IFN $\gamma$-stimulated PD-L1-expressing MC38 cells or (D) PD-L1-expressing CHO cells
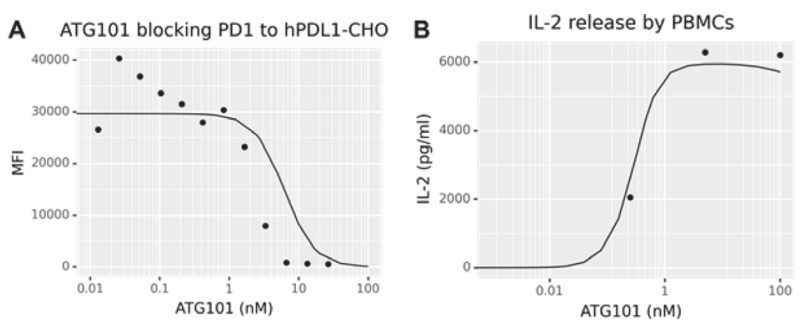

Abstact 227 Figure 3 Comparison of in vitro model simulations to data used for model validation. Lines show simulations, and points show data. (A) PD-1:PD-L1 blockade assay. Reduction in PD-1 binding to PD-L1 at various concentrations of ATG101 was measured on hPDL1-expressing $\mathrm{CHO}$ cells via flow cytometry. (B) IL-2 release assay. PBMCs were activated with staphylococcal enterotoxin A and incubated with ATG101. 


\section{Abstracts}

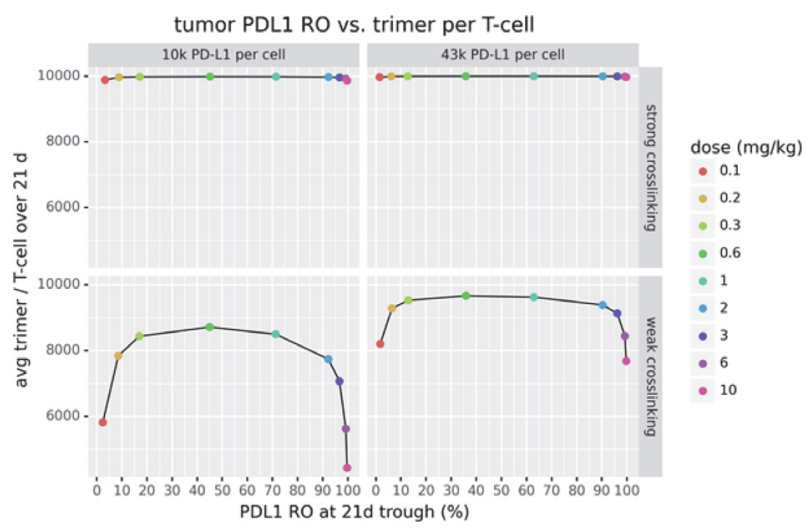

Abstract 227 Figure 4 Trimer formation versus PD-L1 RO at day 21 following a single dose, predicted for different PD-L1 expression levels and crosslinking strengths. Each point represents a dose, with the color indicating the dose level. Panels show predictions for different numbers of PD-L1 receptors per tumor cell (columns) and crosslinking strengths (rows). The $x$ axis indicates the PD-L1 RO at day 21, and the y axis indicates the average number of trimers per T-cell over the 21-day interval following the dose.

Conclusions For bispecific antibodies acting via both receptor crosslinking and checkpoint blockade, reductions in trimer at large doses may complicate dose selection. By predicting PDL1 RO and trimer formation, the model provides a rational basis for clinical dose selection. ATG101 is predicted to be capable of maintaining near-maximal trimer levels across a range of concentrations, allowing it to be dosed at levels high enough to attain greater than 90\% PD-L1 receptor occupancy.

\section{REFERENCES}

1.. Huehls AM, Coupet TA, Sentman CL. Bispecific T-cell engagers for cancer immunotherapy. Immunol Cell Biol 2015;93:290-296

http://dx.doi.org/10.1136/jitc-2021-SITC2021.227 